\section{A Pilot Study to Delineate Factors \\ Contributing to Multi-Drug Resistant Organism (MDRO) Outbreak and Control at the Sangre Grande Hospital}

Rajeev P. Nagassar ${ }^{1}$ Roma J. Bridgelal-Nagassar ${ }^{2}$ Lima Harper ${ }^{1}$ Keston Daniel ${ }^{1}$

Department of Microbiology, Sangre Grande Hospital, ERHA $^{1}$

County Health Administration, St George Central, NWRHA ${ }^{2}$

\section{Correspondence to:}

Dr. Rajeev Nagassar

Department of Micriobiology, Sangre Grande Hospital, ERHA

Email:peeyush3@hotmail.com

\section{ABSTRACT}

\section{BACKGROUND}

The Sangre Grande Hospital (SGH) experienced several Multi Drug Resistant Organism (MDRO) outbreaks for 2015. In this regard, a study was conducted to understand factors contributing to MDRO acquisition and control, for policy and implementation of prevention and control measures.

\section{METHODOLOGY}

A Case-Control Study Design was chosen to execute this research project in order to determine risk factors and factors associated with control of the outbreaks. This was done retrospectively from January to March 2015. Cases and controls were unmatched. Cases were defined as patients admitted to wards with a contracted MDRO, discharged or died and had a medical or surgical diagnosis. MDROs included Methicillin resistant Staphylococcus aureus (MRSA) and Multi Drug Resistant Gram-Negative Bacilli (MDRGNB). Controls were any other patients on the wards with similar demographics and diagnosis and disposition, not infected or colonized with a MDRO and had a medical or surgical diagnosis.

\section{RESULTS}

Eight (8) cases and ten (10) Controls were incorporated in the study. Patients recently hospitalized had 14 times the odds of the group not recently hospitalized, of developing a MDRO, 95\% CI: $1.1352-172.6502, p=0.05$. Length of Stay (LOS) on the ward for a minimum of five days showed a significant association with MDRO acquisition ( $p$ $<0.036)$. The main contributory factors to increased MDRO acquisition: greater than one (1) invasive devices, urinary catheter and/or central venous catheter ( $p<$ 0.043 and $p<0.007$ respectively). Most MDRO cases had at least one invasive device attached to them during their stay on the ward: Central Venous Catheter (CVC), a Urinary Catheter or both. MDRO cases mean LOS: 29.5 days (19.27 S.D.) as opposed to the controls with 5.2 days (4.29 S.D.).

\section{CONCLUSION}

There is a significant association between recent hospitalization and developing an MDRO. Patients with an MDRO also stayed a mean of 29.5 days compared to 5.2 days for controls.

Key Words: MDRO, Case-Control, Invasive Devices, Hospital Exposure 


\section{INTRODUCTION}

Multi-Drug Resistant Organisms (MDROs) pose a challenge to hospitals in terms of treatment and control. ${ }^{1,2}$ These MDROs include Gram positive organisms such as Methicillin Resistant Staphylococcus aureus (MRSA) and multi-drug resistant Gram-negative bacilli (MDRGNB) e.g. Acinetobacter baumannii and Stenotrophomonas maltophilia. These organisms are challenging to treat and control for infection prevention and control departments (IPC). ${ }^{1,2}$ In a paper by Simmons et al., inadequate compliance with hand hygiene and personal protective equipment were factors which promoted poor control of resistant organisms. ${ }^{2}$ These organisms have been identified as pathogens and colonizers with the potential to develop significant drug resistance and cause health care associated infections (HCAI). ${ }^{2}$

In addition, the control of these MDROs is extremely important in decreasing morbidity/mortality and costs in the healthcare setting. It also important in the preservation of the usefulness of antibiotics. ${ }^{1}$ One study evaluated the cost of multi-drug resistant Acinetobacter baumannii (MDRAB). ${ }^{3}$ The study showed that the average total hospital cost of patients who acquired MDRAB was $\$ 98,575$ (USD) higher than that of control patients. ${ }^{3}$ In a study by Qavi et al. patients with ceftazidime-resistant Klebsiella pneumoniae infection experienced increased mortality. ${ }^{4}$ Song et al. have shown that patients with vancomycin resistant Enterococcus spp. stayed in hospital approximately 17 days longer and this organism contributed to significantly excess mortality and economic loss. ${ }^{5}$ It would be interesting to see if resistant organisms result in increased length of stay at the Sangre Grande Hospital (SGH). In addition, there is a general lack of published studies on this data in Trinidad and Tobago. This study will provide novel data to Sangre Grande Hospital and Trinidad and Tobago.

In one systematic review by Tenny et al. factors identified as risk factors for MDR urinary tract infection (UTI) were age greater than 51 years, urinary catheterization, previous hospitalization, previous antibiotics, nursing home residence, gender, previous UTI, and diabetes mellitus. ${ }^{6}$ This study highlighted that several risk factors had minimal or conflicting evidence. This shows the need to delineate risk factors at the SGH in east Trinidad to elucidate risk factors and even factors resulting in prevention and control of MDROs.
The prevalence of just MRSA, for 2015 was 17\%, for the $\mathrm{SGH}$. The SGH would have experienced several MDRO cases at the institution for the year 2015. This would have included several outbreaks. In this regard, the Infection Prevention and Control (IPC) Committee along with hospital management would have instituted several measures to manage and mitigate against such recurrence. One such measure is the establishment and implementation of research for policy development. This study serves to delineate risk factors for multi-drug resistant organism (MDRO) acquisition at the Sangre Grande Hospital (SGH). The study also aims to identify factors necessary for the management of patients with MDROs including which may result in better prevention and control of infection and improved microbiological and clinical outcomes. ${ }^{1}$ This paper focuses on delineating risk factors in a "horizontal" approach to infection prevention and control where risk factors and associated interventions for multiple resistant pathogens are assessed at the SGH and not just one organism as in "vertical" approaches. ${ }^{2}$

\section{METHODOLOGY}

A Case-Control study design was chosen to execute this research project. This was done retrospectively from January to March 2015. Cases and controls were unmatched.

Cases were defined as patients admitted to Wards 2, 3, 5 and Intensive Care (ICU) with a contracted MDRO (colonized or infected) who had a medical or surgical diagnosis and were eventually discharged or died.

Both cases and controls were not strictly matched by sex and ethnic origin. Age was controlled by only selecting age matched cases and controls, from the same wards, between $30-70$ years of age.

MDROs included Methicillin resistant Staphylococcus aureus (MRSA) and multi drug resistant Gram-negative bacilli (MDRGNB) including Acinetobacter baumannii and Stenotrophomonas maltophilia.

The Controls were any other patients on the Wards, with similar demographics and diagnosis, not infected or colonized with a MDRO, had a medical or surgical diagnosis and died or were discharged.

Inclusion criteria: Unmatched patients admitted with a 
diagnosis of infection or carriage of a MDRO, either died or discharged and had a surgical or medical diagnosis.

Exclusion criteria: Patients who were never in one of the wards being investigated or had conditions requiring prolonged stay e.g. traction in Orthopedics and either died or were discharged and had a medical or surgical diagnosis.

The microbiological end point was the actual isolation of an MDRO pathogen or colonizer for cases. The clinical end point was the in-hospital morbidity or mortality for cases.

\section{Data Extraction}

A data extraction sheet was used to extract data from dockets retrospectively for cases and controls. This included dimensions such as demographics and components of a tired system of infection control, including: 1) administrative measures/adherence monitoring 2) MDRO education 3) antimicrobial use 4) surveillance 5) infection control precautions to prevent transmission and 6) environmental measures. Specifically, the following areas were examined: presenting symptoms and signs, investigations performed, data in the history of the complaint, management data, data specific to education, cleaning and use of personal protective equipment, communication and final disposal of the cases and controls.

Measures were taken to ensure data was captured anonymously and all data was protected on a password protected computer and in a locked cupboard.

\section{Statistical Analysis}

The statistical analysis was with IBM SPSSv22. The $\mathrm{Chi}^{2}$ test or $\mathrm{t}$ - Test, for small samples, were the tests of significance. The t-Test was used where a $2 \times 2$ table could not be used. The Fisher's exact test was used for 2 $\times 2$ tables with small sample sizes. Tests of significance were 2 tailed. A p value of $<0.05$ was considered significant. Odds ratios were used where the 2 by 2 table allowed. If odds ratios could not be calculated the significance value or $p$ value, of $<0.05$ was used.

\section{Ethical Approval}

The ethics committee of the Eastern Regional Health Authority approved this study.

\section{RESULTS}

Eight (8) cases and ten (10), unmatched, controls were surveyed during the data collection phase of the project. Ages ranged from 30 to 60 years. There were 4 males and 14 females. 17 lived in rural areas while 1 lived in an urban area.

Patients who were hospitalized before the current admission, had approximately 14 times the odds of those not recently hospitalized (prior to the current admission) group of developing a MDRO ( 95\% CI: $1.1352-$ $172.6502, p=0.05$ ) All patients, both cases and controls, returned home following their hospitalization. There were no deaths. They were all cleared of the MDRO before leaving.

\section{Contributory Factors for contraction of an MDRO Colonization or Infection:}

If more than one (1) Invasive Device was being used by the patient, particularly Urinary Catheter and/or Central Venous Catheter ( $p<0.043$ and $p<0.007$ respectively), this was significantly associated with contacting an MDRO. All MDRO cases except one (1), had at least one other invasive device attached to them during their stay on the ward (with that device either being a CVC, a Urinary/Catheter or both). Usage of multiple invasive devices was associated with MDRO $(p<0.001)$. This is illustrated in table 1.

A length of stay on the ward for greater than five (5) days, showed a significant association with MDRO acquisition ( $p<0.036)$. This is shown in table 2 . Cases who contracted an MDRO had a longer length of stay overall compared to controls, with the mean length of stay of MDRO cases being 29.5 days (19.27 S.D.) as opposed to the controlled patients being on the Ward for an average of 5.2 days ( 4.29 S.D.).

\section{Presenting Symptom}

No statistically significant association was uncovered that pointed to any specific presentation symptom and sign that related to a MDRO infection or colonization, in this study.

\section{Investigations}

Investigations significantly associated with an MDRO acquisition included, arterial blood gas, $\mathrm{p}<0.007$ and the 
Table 1. Association between the use of Invasive Devices and the Contraction of a MDRO

\begin{tabular}{|l|c|c|c|}
\hline & Cases & Controls & \multirow{2}{*}{} \\
\cline { 2 - 4 } & $\#$ & $\#$ & \multirow{2}{*}{ *p value } \\
\hline IV** & 8 & 8 & 0.477 \\
\hline Urinary Catheter & 5 & 1 & $\underline{\mathbf{0 . 0 4 3}}$ \\
\hline CVC*** & 5 & 0 & $\underline{\mathbf{0 . 0 0 7}}$ \\
\hline NGT**** & 2 & 0 & 0.183 \\
\hline Usage Of Multiple Invasive Devices & 8 & 1 & $\underline{\mathbf{0 . 0 0 1}}$ \\
\hline
\end{tabular}

**Intravascular line $\quad * * *$ Central Venous Catheter $\quad * * * *$ Nasogastric Tube

MDRO - Multi Drug Resistant Organism

Table $2.2 \times 2$ table used to show the number of patients with LOS greater or less than 5 days and the risk of contraction of a MDRO

\begin{tabular}{|l|c|c|}
\hline Length Of Stay (LOS) $* *$ & Cases & Controls \\
\hline LOS** over 5 days (\# of Patients) & 8 & 5 \\
\hline LOS 5 days or less (\# of Patients) & 0 & 5 \\
\hline
\end{tabular}

*Fisher's Exact Test: $\mathrm{p}<0.035 \quad$ ** Length of Stay

hemoglobin parameter in the complete blood count $(\mathrm{CBC})$, $p<0.015$, specifically anemia. The patient management factor significantly associated with clearing the MDRO was culture directed antibiotics, $\mathrm{p}<0.001$.

\section{Infection Prevention and Control Factors}

Environmental cleaning, $\mathrm{p}<0.015$ and barrier nursing, $\mathrm{p}$ $<0.015$ were all significantly and dedicated staff and equipment, $\mathrm{p}<0.025$ associated with clearing the MDRO.

\section{Communication}

Communication between IPC and management, between IPC and microbiology and communication between IPC and the Ward, education between IPC and Ward staff, ongoing education of cleaning staff, all had no associated with a positive outcome of clearing of the MDROs. However, the IPC intervention of education was significantly associated with clearance of the MDRO, $p<$ 0.015 .

Odds Ratios for some of the indicators could not be calculated since there was a nil value in the $2 \times 2$ table used to determine these proportions.

\section{DISCUSSION}

The odds of a patient being recently hospitalized versus a patient who was not recently hospitalized and acquiring an MDRO was 14. It is important to note that both cases and controls were hospitalized patients, however cases had a higher odds of having hospitalization prior to the current stay, the length of that hospitalization was not determined. It should be noted that the confidence interval did not include 1 and thus is significant but the Fisher's Exact Test showed that the association was insignificant, but just at 0.05 . Previous hospitalization or continuous exposure to the healthcare environment probably predisposes persons at increased risk of being exposed to MDROs at the clinics or wards. Thus, this points to the fact that recent hospitalization, prior to the current stay, increases the odds of MDRO acquisition (colonization or infection) at the SGH. This has been highlighted in a study by Gross et al. which showed that the odds of multidrug resistance increased when admission from a healthcare facility, such as a nursing home, was considered. ${ }^{7}$

In addition, in this study, the length of stay of cases with an MDRO was almost 30 days for cases compared to five 
(5) days for controls. This is similar to the 17 day longer stay noted by Song et al. for patients with an MDRO, 12 days of which were in the intensive care unit. This implicates recent exposure to the healthcare environment increasing the odds of acquiring an MDRO and increasing the duration of hospitalization, as seen in this study and other studies. 5

It should be noted that there was a statistically significant association of developing an MDRO, with more than one invasive device, particularly urinary catheters and central venous lines. This has been shown in studies by Razaviab et $a l^{8}$ They showed a significant association between prolonged urinary catheterization and both nosocomial infections and deaths. ${ }^{8}$ In addition, Hao-Yuan et al. have shown that increased frequency of venipunctures, especially when there was no concurrent antibiotic use, substantially raised the risk of central line associated blood stream infection. ${ }^{9}$ Invasive devices were used for patients in both cases and control categories. There was an association between multiple invasive devices being used and the increased risk of contraction of a MDRO like that highlighted in other studies. ${ }^{1,8-9}$

Hemoglobin may have fallen due to the increased length of stay of almost one month. In a study by Ballo et a.l, colonized versus non-colonized patients had similar hemoglobin levels throughout the hospital stay, in contrast to this study. ${ }^{10}$ Arterial blood gases are usually done in very sick patients, as in some of the cases in this study. This may account for the significant association. In a study by Aliberti et al. it was found that patients with MDRO risk factors were significantly more likely to have lower hemoglobin counts and lower partial pressure of oxygen in their arterial blood gas, this is similar to this study, although this study did not focus on respiratory tract infections as Aliberti et al. ${ }^{11}$

Culture directed and appropriate use of antibiotics are expected to improve microbial and clinical outcome, if given in the correct time and appropriately. ${ }^{1,10-11}$ Environmental cleaning and proper use of standard and additional precautions are well known to assist with clearing MDROS, as shown in this study. ${ }^{1,2}$ Lastly communication is important in any organization in achieving good outcomes, even though most studies did not show a significant association with clearing the
MDROs.

In this study, we saw that educational interventions, such as implementing staff training can promote effective control of infection or colonization, as recommended by Siegel et al. ${ }^{1}$. Thus, similar to the multifaceted interventions proposed by Siegel et al., this study shows that use of administrative measures/adherence monitoring, MDRO education, judicious/appropriate antimicrobial use, surveillance, infection control precautions (to prevent transmission) and environmental measures can all work together to control MDRO transmission at the SGH in Trinidad and Tobago. ${ }^{1}$

It should be noted that case-control studies are good studies for determining risk factors. ${ }^{12}$ This type of study usually presents odds ratio which are useful for multiple exposures and rare diseases. There is also less potential for loss to follow up, useful for short term and for small sample of cases, as in this study. Case-control studies, however, are potentially weaker causal investigations, there is more potential for recall bias and the study may not be generalizable but good for use at the SGH in east Trinidad. It does not allow examination of natural course of disease, survival. A bias could have occurred by not strictly matching cases and controls. ${ }^{13}$ Another weakness of this study is that it does not distinguish between infection and colonization. This last point can also be a strength as this study can be applied to both infection and colonization and thus to multiple MDRO control, or horizontal strategies, in general at the $\mathrm{SGH}^{2}$

\section{CONCLUSION}

This study highlights the importance of understanding factors that put patients at risk of infections/colonization and understanding prevention and control strategies. It helps in the control of MDROs and reducing increased length of stay in hospital and thus hospital costs. More research is needed in defining risk factors for "horizontal' and "vertical' infection prevention and control measures. ${ }^{1,2}$ Finally it should be noted that this was a pilot study and thus our findings need further investigation and/or further study. 
Ethical approval statement: the study was approved by the Eastern Regional Health Authority

Informed consent: not applicable

Funding: none

Conflicts of interest: none to declare

Author contributions: RP Nagassar and RJ Bridgelal Nagassar conceived paper, oversaw data collection, wrote manuscript, statistical analysis and approved the final version. L. Harper oversaw data collection and K Daniel oversaw design and data analysis.

\section{REFERENCES}

1. Siegel, JD, Rhinehart $E$, Jackson $M$ et al. Management of multidrug-resistant organisms in health care settings. Am J Infect Control. 2007; 35(10):S165-93.

2. Simmons BP, Larson EL. Multiple drug resistant organisms in healthcare: the failure of contact precautions. J Infect Prev. 2015;16(4):178-181.

3. Wilson SJ, Knipe CJ, Zieger MJ et al. Direct costs of multidrug-resistant Acinetobacter baumannii in the burn unit of a public teaching hospital. Am J Infect Control. 2004 Oct;32(6): 342-4.

4. Qavi A1, Segal-Maurer S, Mariano N et al. Increased mortality associated with a clonal outbreak of ceftazidime-resistant Klebsiella pneumoniae: a casecontrol study. Infect Control Hosp Epidemiol. 2005; 26 (1): 63-8.

5. Song X1, Srinivasan A, Plaut D, Perl TM. Effect of nosocomial vancomycin-resistant enterococcal bacteremia on mortality, length of stay, and costs. Infect Control Hosp Epidemiol. 2003 ;24(4):251-6.

6. Tenneya J, Hudson N, Alnifaidy H, Ting Cheung Li J, Harriet Fung K. Risk factors for acquiring multidrugresistant organisms in urinary tract infections: $A$ systematic literature review. Saudi Pharm J. 2018; 26 (5): 678-684.

7. Gross AE, Van Schooneveldd TC, Olsenc KM. et al. Epidemiology and Predictors of Multidrug-Resistant Community-Acquired and Health Care-Associated Pneumonia. Antimicrob. Agents Chemother. 2014 ;58 (9): 5262-5268.

8. Razaviab SM, Dabirana S, Ataeia A, Meysamiea A. Prolonged urinary catheterization, a factor associated with intensive care infections and deaths. Egypt. J. Crit. Care Med. 2014; 2 (1): 37-41.
9. Hao-Yuan C, Chun-Yi L, Li-Min H, Ping-Ing L, Jong-Min $C$, Luan-Yin $C$. Increased frequency of peripheral venipunctures raises the risk of central-line associated bloodstream infection in neonates with peripherally inserted central venous catheters. J Microbiol Immunol Infect. 2016; 49 (2): 230-236.

10.Ballo O, Tarazzit I, Stratmann J, et al. Colonization with multidrug resistant organisms determines the clinical course of patients with acute myeloid leukemia undergoing intensive induction chemotherapy. PLOS One. 2019; 14(1):e0210991. Published 2019 Jan 23. doi:10.1371/journal.pone.0210991

11.Aliberti S, Di Pasquale M, Zanaboni AM, Cosentini R, Brambilla AM, Seghezzi S et al. Stratifying Risk Factors for Multidrug-Resistant Pathogens in Hospitalized Patients Coming From the Community With Pneumonia. Clin Infect Dis. 2012; 470-478.

12.Ikram R, Psutka R, Carter A, Priest P. An outbreak of multi-drug resistant Escherichia coli urinary tract infection in an elderly population: a case-control study of risk factors. BMC Infect. Dis. 201515:224. Available from: https://doi.org/10.1186/s12879-015-0974-0.

13.Lewallen S, Courtright P. Epidemiology in Practice: Case-Control Studies. Community Eye Health. 1998; 11 (28):57-58. 